\title{
Empirische Forschung zur Wirksamkeit von Führungsverhalten in Nordamerika
}

Was ist gute Führung und wie sollte sich eine Führungskraft verhalten? Bis in die 50er Jahre schien diese Frage leicht zu beantworten zu sein. Es herrschte der Glaube vor, dass es sich bei guter Führung um eine angeborene Eigenschaft handle. Man nahm und nimmt auch heute stellenweise noch an, dass sich geborene charismatische Führer im Sinne Max Webers von selbst im Laufe der Zeit durchsetzen. Von George Washington bis Steve Jobs werden „große Führer“ bis heute als Erfolgsgarant für Organisationen angesehen. Die Schulung einer solchen Führungskraft wäre erfolglos, da es sich ja um eine pränatale Prägung handelt.

Diesem Denken widersprachen in der US-amerikanischen Literatur Fleishman und Rensis Likert mit den Ohio State Leadership Studies und den Michigan Leadership Studies. Sie wollten erfolgreiche Führungsstile wissenschaftlich evaluieren. Aufbauend auf den Ergebnissen dieser beiden Studien versuchten Blake und Mouton 1964 aufgabenorientierte und mitarbeiterorientierte Führungsstile mithilfe des sogenannten Managerial Grids zu kartografieren, um gute Führung lernbar zu machen. Führung entwickelte sich zu einem mehrdimensionalen Konzept, das an bestimmte Verhaltensweisen gebunden und durchaus erlernbar ist. ${ }^{1}$

Die Wirkung verschiedener Führungsstile auf den Erfolg einer Organisation ist Gegenstand vieler Studien. Aufgrund mangelnder prognostischer Validität konnte sich in der Theorie bis heute kein Führungsstil herausstellen, der den Anspruch hegt, der ,,richtige“ zu sein. Die mangelnde Validität fußt auf mehreren methodischen wie inhaltlichen Problemen. Es ist nicht möglich, die Effektivität von guter Führung zu messen, ohne die Motivation des Mitarbeiters zu betrachten. Diese wird jedoch von verschiedenen intrinsischen und extrinsischen Faktoren mitbeeinflusst.

${ }^{1}$ Vgl. Yukl (2010).

() Springer Fachmedien Wiesbaden GmbH 2017

L. Bartosch et al., Ziel- und Leistungsorientierung, essentials, DOI 10.1007/978-3-658-16882-7_1 